\title{
Pairwise mutual knowledge and correlated rationalizability
}

Citation for published version (APA):

Tsakas, E. (2012). Pairwise mutual knowledge and correlated rationalizability. METEOR, Maastricht University School of Business and Economics. METEOR Research Memorandum No. 031 https://doi.org/10.26481/umamet.2012031

Document status and date:

Published: 01/01/2012

DOI:

10.26481/umamet.2012031

Document Version:

Publisher's PDF, also known as Version of record

\section{Please check the document version of this publication:}

- A submitted manuscript is the version of the article upon submission and before peer-review. There can be important differences between the submitted version and the official published version of record.

People interested in the research are advised to contact the author for the final version of the publication, or visit the DOI to the publisher's website.

- The final author version and the galley proof are versions of the publication after peer review.

- The final published version features the final layout of the paper including the volume, issue and page numbers.

Link to publication

\footnotetext{
General rights rights.

- You may freely distribute the URL identifying the publication in the public portal. please follow below link for the End User Agreement:

www.umlib.nl/taverne-license

Take down policy

If you believe that this document breaches copyright please contact us at:

repository@maastrichtuniversity.nl

providing details and we will investigate your claim.
}

Copyright and moral rights for the publications made accessible in the public portal are retained by the authors and/or other copyright owners and it is a condition of accessing publications that users recognise and abide by the legal requirements associated with these

- Users may download and print one copy of any publication from the public portal for the purpose of private study or research.

- You may not further distribute the material or use it for any profit-making activity or commercial gain

If the publication is distributed under the terms of Article $25 \mathrm{fa}$ of the Dutch Copyright Act, indicated by the "Taverne" license above, 


\section{Maastricht University}

Elias Tsakas

Pairwise Mutual Knowledge and Correlated Rationalizability

RM/12/031

\section{METEOR}

Maastricht University School of Business and Economics

Maastricht Research School of Economics

of Technology and Organization

P.O. Box 616

NL - 6200 MD Maastricht

The Netherlands 


\title{
PAIRWISE MUTUAL KNOWLEDGE AND CORRELATED RATIONALIZABILITY ${ }^{1}$
}

\section{ELIAS TSAKAS}

\begin{abstract}
We provide epistemic conditions for correlated rationalizability, which are considerably weaker than the ones by Zambrano (2008). More specifically, we simultaneously replace mutual knowledge of rationality and mutual knowledge of the event that every player deems possible only strategy profiles that belong to the support of her actual conjecture, with strictly weaker epistemic conditions of pairwise mutual knowledge of these events. Moreover, we show that our epistemic foundation for correlated rationalizability does not imply mutual knowledge of rationality.
\end{abstract}

KEYWORDS: Correlated rationalizability, pairwise mutual knowledge, rationality, conjectures, epistemic game theory.

\section{INTRODUCTION}

Rationalizability was independently introduced by the seminal papers of Bernheim (1984) and Pearce (1984). Soon after that, Brandenburger and Dekel (1987) defined the slightly more general concept of correlated rationalizability $^{1}$ which allows players to hold correlated beliefs about the opponents' strategy profile. Correlated rationalizability was quickly recognized as one of the central solution concepts in noncooperative game theory, as it yields the strategy profiles that survive iterated elimination of strictly dominated strategies (Böge and Eisele, 1979; Tan and Werlang, 1988; Brandenburger and Dekel, 1987).

According to the standard epistemic foundations, correlated rationalizability is characterized by common knowledge ${ }^{2}$ of rationality (Böge and Eisele, 1979; Tan and Werlang, 1988; Brandenburger and Dekel, 1987). Recently, Zambrano (2008) provided sufficient conditions for correlated rationalizability that do not involve common knowledge of rationality. In fact, he showed that if it is mutual knowledge that all players are rational, and also it is mutual knowledge that every player deems possible only strategy profiles that belong to support of the actual conjecture, then a correlated rationalizable strategy profile is played. The idea behind this result is similar to the standard epistemic conditions for Nash

\footnotetext{
${ }^{1}$ I am indebted to Christian Bach for fruitful discussions on this paper.

${ }^{1}$ Actually, this idea was already present in Böge and Eisele (1979). However, this paper has been slightly overlooked, mainly due to the fact that they used somewhat non-standard terminology and notation. For an overview of this literature we refer to Perea (2012).

${ }^{2}$ In the literature, knowledge and probability-1 belief are often considered synonyms. In fact, the only difference between the two notions is that knowledge requires the truth axiom, i.e., if something is known then it is necessarily true. For many solution concepts, such as correlated rationalizability or Nash equilibrium, making the distinction is not necessary. In this paper, we adopt the standard set theoretic definition of knowledge á la Aumann (1976).
} 
equilibrium by Aumann and Brandenburger (1995), in that it places restrictions not only on rationality but also on the players' level knowledge about other players' conjectures.

In a recent paper, Bach and Tsakas (2012) refined Aumann and Brandenburger's epistemic conditions for Nash equilibrium by simultaneously replacing common knowledge of conjectures and mutual knowledge of rationality, with pairwise common knowledge of conjectures and pairwise mutual knowledge of rationality for only some pairs of players respectively. In this paper, we similarly introduce weaker epistemic conditions for correlated rationalizability than those by Zambrano (2008), by simultaneously relaxing mutual knowledge of rationality and mutual knowledge every player deeming possible only strategy profiles that belong to support of the actual conjecture. Our new conditions are based on imposing pairwise mutual knowledge of rationality and pairwise mutual knowledge of every player deeming possible only strategy profiles that belong to support of the actual conjecture only for some pairs of players. This weakening becomes very significant in games with a large number of players. Finally, we show that our conditions do not imply mutual knowledge of rationality. To our knowledge, this is the first paper in the literature to provide epistemic conditions for correlated rationalizability without mutual knowledge of rationality.

\section{PRELIMINARIES}

\subsection{Normal form games}

Consider a normal form game $\Gamma=\left(N,\left(S_{i}\right)_{i \in N},\left(U_{i}\right)_{i \in N}\right)$, where $N=\{1, \ldots, n\}$ denotes the finite set of players with typical elements $i$ and $j$, and $S_{i}$ is the finite set of pure strategies with typical element $s_{i}$ for every player $i \in I$. As usual, define $S:=S_{1} \times \cdots \times S_{n}$ with typical element $s=\left(s_{1}, \ldots, s_{n}\right)$ and $S_{-i}:=S_{1} \times \cdots \times S_{i-1} \times S_{i+1} \times \cdots \times S_{n}$ with typical element $s_{-i}=\left(s_{1}, \ldots, s_{i-1}, s_{i+1}, \ldots, s_{n}\right)$. The function $U_{i}: S_{i} \times S_{-i} \rightarrow \mathbb{R}$ denotes player $i$ 's payoff function.

A probability measure $\mu_{i} \in \Delta\left(S_{-i}\right)$ on the set of the opponents' strategy profiles is called a conjecture of $i$, with $\mu_{i}\left(s_{-i}\right)$ signifying the probability that $i$ attributes to the opponents playing $s_{-i}$. Slightly abusing notation, let $\mu_{i}\left(s_{j}\right)$ denote the probability that $i$ assigns to $j$ playing $s_{j}$, i.e., $\mu_{i}\left(s_{j}\right)$ is the probability that $\operatorname{marg}_{S_{j}} \mu_{i}$ attaches to $s_{j}$. As usual we allow for correlated beliefs, i.e. $\mu_{i}$ is not necessarily a product measure, hence the probability $\mu_{i}\left(s_{1}, \ldots, s_{i-1}, s_{i+1}, \ldots, s_{n}\right)$ can differ from the product $\prod_{j \neq i} \mu_{i}\left(s_{j}\right)$ of the marginal probabilities ${ }^{3}$. Given a conjecture $\mu_{i} \in \Delta\left(S_{-i}\right)$, player $i$ 's expected payoff from playing

\footnotetext{
${ }^{3}$ Intuitively, a player's belief on his opponents' choices can be correlated, even though players choose their strategies independently from each other.
} 
some strategy $s_{i} \in S_{i}$ is given by

$$
u_{i}\left(s_{i}, \mu_{i}\right):=\sum_{s_{-i} \in S_{-i}} \mu_{i}\left(s_{-i}\right) U_{i}\left(s_{i}, s_{-i}\right)
$$

We say that a strategy $s_{i}$ is a best response to $\mu_{i}$, and write $s_{i} \in B R_{i}\left(\mu_{i}\right)$, whenever $u_{i}\left(s_{i}, \mu_{i}\right) \geq u_{i}\left(s_{i}^{\prime}, \mu_{i}\right)$ for all $s_{i}^{\prime} \in S_{i}$.

For each $i \in N$, consider some $B_{i} \subseteq S_{i}$. Then, we say that the rectangle $B_{1} \times \cdots \times B_{n} \subseteq S$ satisfies the best response property whenever for each $s_{i} \in B_{i}$ there exists some $\mu_{i} \in \Delta\left(B_{-i}\right)$ with $s_{i} \in B R_{i}\left(\mu_{i}\right)$ (Brandenburger and Dekel, 1987, Def. 2.1). Notice that the previous definition of the best response property differs from the one by Pearce (1984, Def. 2) in that Pearce requires that for every $s_{i}$ the conjecture $\mu_{i}$ with $s_{i} \in B R_{i}\left(\mu_{i}\right)$ is such that the marginal distributions are independent. Throughout the present paper the term best response property refers to the definition by Brandenburger and Dekel (1987).

A strategy profile $\left(s_{1}, \ldots, s_{n}\right)$ is said to be correlated rationalizable whenever there is some $B_{1} \times \cdots B_{n}$ satisfying the best response property such that $\left(s_{1}, \ldots, s_{n}\right) \in B_{1} \times \cdots \times B_{n}$. It is well-known that a strategy profile is correlated rationalizable if and only if it survives iterated elimination of strictly dominated strategies.

\subsection{Epistemic Models}

Unlike classical game theory which is based on two basic primitives — strategies and payoffs epistemic game theory explicitly models conjectures as a third basic element, thus adding an epistemic framework to the description and analysis of the game. Notice that within the epistemic framework often we also model beliefs about the payoff functions, thus allowing for incomplete information games (e.g., Aumann and Brandenburger, 1995; Zambrano, 2008). In this paper, without of loss of generality for our results, we only consider complete information games, i.e., payoff functions are common knowledge.

Recall the standard partitional model introduced by Aumann (1976): Formally, an epistemic model of some game $\Gamma$ is a tuple $\mathcal{A}^{\Gamma}=\left(\Omega,\left(\pi_{i}\right)_{i \in N},\left(\mathcal{P}_{i}\right)_{i \in N},\left(\hat{s}_{i}\right)_{i \in N}\right)$, consisting of a finite set $\Omega$ of states with typical element $\omega$, together with a full support prior $\pi_{i} \in \Delta(\Omega)$ for each $i \in N$. Every player $i \in N$ is endowed with an information partition $\mathcal{P}_{i}$ of $\Omega$, with $P_{i}(\omega)$ denoting the element of $\mathcal{P}_{i}$ that contains $\omega$ : It is the set of states that $i$ deems possible at $\omega$. For each event $E \subseteq \Omega$, we define the set of states where $i$ knows $E$ by

$$
K_{i}(E):=\left\{\omega \in \Omega: P_{i}(\omega) \subseteq E\right\}
$$


An event is mutually known at some state if everyone knows it. Formally, we say that $E$ is mutual knowledge at $\omega$, whenever $\omega \in K(E)$, where

$$
K(E):=\bigcap_{i \in N} K_{i}(E) .
$$

For every player $i \in N$, the function $\hat{s}_{i}: \Omega \rightarrow S_{i}$ specifies $i$ 's strategy at each state. As usual, we assume that $\hat{s}_{i}$ is $\mathcal{P}_{i}$-measurable, i.e. $\hat{s}_{i}\left(\omega^{\prime}\right)=\hat{s}_{i}(\omega)$ for all $\omega^{\prime} \in P_{i}(\omega)$, implying that $i$ knows her own strategy. Moreover, let $\hat{s}_{-i}(\omega):=\left(\hat{s}_{1}(\omega), \ldots, \hat{s}_{i-1}(\omega), \hat{s}_{i+1}(\omega), \ldots, \hat{s}_{n}(\omega)\right)$.

Given an epistemic model, the function $\hat{\mu}_{i}: \Omega \rightarrow \Delta\left(S_{-i}\right)$ specifies each player's conjecture at every state by attaching to each $s_{-i} \in S_{-i}$ probability

$$
\hat{\mu}_{i}(\omega)\left(s_{-i}\right):=\pi_{i}\left(\left\{\omega^{\prime} \in \Omega: \hat{s}_{-i}\left(\omega^{\prime}\right)=s_{-i}\right\} \mid P_{i}(\omega)\right)
$$

Obviously, $\hat{\mu}_{i}$ is $\mathcal{P}_{i}$-measurable, i.e., $\hat{\mu}_{i}\left(\omega^{\prime}\right)=\hat{\mu}_{i}(\omega)$ for all $\omega^{\prime} \in P_{i}(\omega)$, implying that $\hat{\mu}_{i}$ induces a coarsening of $\mathcal{P}_{i}$. Throughout the paper, for an arbitrary $B_{-i} \subseteq S_{-i}$, let

$$
C_{i}\left(B_{-i}\right):=\left\{\omega \in \Omega: \operatorname{Supp}\left(\hat{\mu}_{i}(\omega)\right) \subseteq B_{-i}\right\}
$$

denote the states where $i$ deems possible only opponents' strategy profiles belonging to $B_{-i}$.

Player $i$ is rational at some state $\omega$, whenever $\hat{s}_{i}(\omega) \in B R_{i}\left(\hat{\mu}_{i}(\omega)\right)$. Let

$$
R_{i}:=\left\{\omega \in \Omega: \hat{s}_{i}(\omega) \in B R_{i}\left(\hat{\mu}_{i}(\omega)\right)\right\}
$$

denote the event that $i$ is rational. Finally, let $R:=\bigcap_{i \in N} R_{i}$.

\subsection{Epistemic conditions for correlated rationalizability}

According to the standard epistemic characterization of correlated rationalizability, a strategy profile is correlated rationalizable if and only if it can be rationally played under common knowledge of rationality (Böge and Eisele, 1979; Brandenburger and Dekel, 1987; Tan and Werlang, 1988). In a recent paper, Zambrano (2008) provided alternative epistemic conditions ${ }^{4}$ that do not involve common knowledge of rationality. More specifically, he showed that if at some state it is mutually known that every player is rational, and also that every player attaches positive probability only to strategy profiles that belong to the support of her actual conjecture, then a correlated rationalizable strategy is played.

\footnotetext{
${ }^{4}$ Zambrano (2008), as well as previous papers in the literature such as Aumann and Brandenburger (1995), employ the formalism of type structures, which is notationally different, but still formally equivalent to the partitional model used in this paper.
} 
TheOREM 1 (Zambrano, 2008) Let $\Gamma$ be a normal form game and $\mathcal{A}^{\Gamma}$ an epistemic model of it. Suppose that there is some state $\omega \in \Omega$ such that $\omega \in K(R) \cap K\left(\bigcap_{i \in N} C_{i}\left(\operatorname{Supp}\left(\hat{\mu}_{i}(\omega)\right)\right)\right)$. Then,

$$
\left(\bigcup_{j \neq 1} \operatorname{Proj}_{S_{1}} \operatorname{Supp}\left(\hat{\mu}_{j}(\omega)\right)\right) \times \cdots \times\left(\bigcup_{j \neq n} \operatorname{Proj}_{S_{n}} \operatorname{Supp}\left(\hat{\mu}_{j}(\omega)\right)\right)
$$

satisfies the best response property, and therefore $\left(\hat{s}_{1}(\omega), \ldots, \hat{s}_{n}(\omega)\right)$ is correlated rationalizable.

In the next section, we provide weaker sufficient conditions for correlated rationalizability, in that we simultaneously substitute mutual knowledge with pairwise mutual knowledge only for some pairs of players, similarly to the way Bach and Tsakas (2012) weaken Aumann-Brandenburger's standard epistemic conditions for Nash equilibrium.

\section{PAIRWISE MUTUAL KNOWLEDGE}

By definition, an event becomes mutually known if it is announced to every player. Yet, an event may be announced to some but not all players. For instance, this is the case if it is announced to Ann and Bob, but not to Carol. Pairwise mutual knowledge of the event between Ann and Bob would then emerge, but not necessarily mutual knowledge. Due to such epistemic possibilities we now recall the pairwise mutual knowledge operator, which was first introduced in Bach and Tsakas (2012).

Let $E \subseteq \Omega$ be some event and $i, j \in N$ be two players. We say that $E$ is pairwise mutual knowledge between $i$ and $j$ whenever they both know $E$. Formally, pairwise mutual knowledge of $E$ between $i$ and $j$ is denoted by the event

$$
K_{i, j}(E):=K_{i}(E) \cap K_{j}(E) .
$$

Note that mutual knowledge implies pairwise mutual knowledge, but not conversely.

In contrast to the standard notion of mutual knowledge, our pairwise epistemic operator describes mutual knowledge only locally for pairs of agents, postulating the existence of exclusively binary relations of epistemic relevance. Formally, we represent a set of such binary relations by means of an undirected graph $G=(N, \mathcal{E})$, where the set of vertices $N$ denotes the set of players from $\Gamma$, and the set of edges $\mathcal{E}$ describe binary symmetric relations $(i, j) \in N \times N$ between pairs of players.

The graph $G$ does neither enrich the epistemic model nor add any additional structure to the game whatsoever, but only provides a formal framework for expressing pairwise local conditions of mutual knowledge, e.g. a graph containing an edge between $i$ and $j$ but not between $j$ and $k$ can be used to model a situation where an event is pairwise mutual knowledge between $i$ and $j$ but not between $j$ and $k$. Thus, the connectedness of two agents by an edge is of purely epistemic and not physical 
character. However, $G$ could also be interpreted as a network. For instance, in a large economy agents may have access to information about relevant personal characteristics — such as rationality — of their neighbours only.

Next, some graph theoretic notions are recalled. A sequence $\left(i_{k}\right)_{k=1}^{m}$ of players is a path whenever $\left(i_{k}, i_{k+1}\right) \in \mathcal{E}$ for all $k \in\{1, \ldots, m-1\}$, i.e. in a path every two consecutive players are linked by an edge. Moreover, a graph $G$ is called connected if it contains a path $\left(i_{k}\right)_{k=1}^{m}$ such that for every $i \in N$ there is some $k \in\{1, \ldots, m\}$ with $i_{k}=i$. In addition, $G$ is complete, if $(i, j) \in \mathcal{E}$ for all $i, j \in N$.

Two specific pairwise-local mutual knowledge conditions are now introduced.

Definition 1 Let $\Gamma$ be a game, $\mathcal{A}^{\Gamma}$ be an epistemic model of it, $G$ be an undirected graph, and $\omega$ be a state.

$\bowtie$ Rationality is $G$-pairwise mutual knowledge at $\omega$, whenever $\omega \in K_{i, j}\left(R_{i} \cap R_{j}\right)$ for all $(i, j) \in \mathcal{E}$.

$\otimes$ It is G-pairwise mutual knowledge at $\omega$ that players deem possible only strategy profiles belonging to the actual support of their conjectures, whenever $\omega \in K_{i, j}\left(C_{i}\left(\operatorname{Supp}\left(\hat{\mu}_{i}(\omega)\right)\right) \cap C_{j}\left(\operatorname{Supp}\left(\hat{\mu}_{j}(\omega)\right)\right)\right)$ for all $(i, j) \in \mathcal{E}$.

Note that henceforth an edge between two agents $i$ and $j$ in a graph $G$ signifies that $i$ and $j$ entertain pairwise mutual knowledge of rationality as well as pairwise mutual knowledge of everybody deeming possible only strategy profiles belonging to the actual support of their conjectures.

The epistemic conditions introduced in Definition 1 are clearly weaker than the ones used by Zambrano (2008). Formally, observe that

$$
\begin{aligned}
K(R) & =\bigcap_{i \in N} K_{i}\left(R_{1} \cap \cdots \cap R_{n}\right) \\
& \subseteq \bigcap_{i \in N} \bigcap_{j \in N:(i, j) \in \mathcal{E}} K_{i, j}\left(R_{1} \cap \cdots \cap R_{n}\right) \\
& \subseteq \bigcap_{i \in N} \bigcap_{j \in N:(i, j) \in \mathcal{E}} K_{i, j}\left(R_{i} \cap R_{j}\right),
\end{aligned}
$$

while at the same time

$$
\begin{aligned}
\left.K\left(\bigcap_{i \in N} C_{i}\left(\operatorname{Supp}\left(\hat{\mu}_{i}(\omega)\right)\right)\right)\right) & =\bigcap_{i \in N} K_{i}\left(C_{1}\left(\operatorname{Supp}\left(\hat{\mu}_{1}(\omega)\right)\right) \cap \cdots \cap C_{n}\left(\operatorname{Supp}\left(\hat{\mu}_{n}(\omega)\right)\right)\right) \\
& \subseteq \bigcap_{i \in N} \bigcap_{j \in N:(i, j) \in \mathcal{E}} K_{i, j}\left(C_{1}\left(\operatorname{Supp}\left(\hat{\mu}_{1}(\omega)\right)\right) \cap \cdots \cap C_{n}\left(\operatorname{Supp}\left(\hat{\mu}_{n}(\omega)\right)\right)\right) \\
& \subseteq \bigcap_{i \in N} \bigcap_{j \in N:(i, j) \in \mathcal{E}} K_{i, j}\left(C_{i}\left(\operatorname{Supp}\left(\hat{\mu}_{i}(\omega)\right)\right) \cap C_{j}\left(\operatorname{Supp}\left(\hat{\mu}_{j}(\omega)\right)\right)\right) .
\end{aligned}
$$

Observe that in our context mutual knowledge coincides with $G$-pairwise mutual knowledge whenever $G$ is complete. 
The following example illustrates the two new concepts of $G$-pairwise mutual knowledge and also relates them to the standard notions of mutual knowledge as used by Zambrano (2008).

EXAmple 1 Consider the symmetric normal form game $\Gamma=\left(N,\left(S_{i}\right)_{i \in N},\left(U_{i}\right)_{i \in N}\right)$, where $N=$ $\{$ Ann $(a)$, Bob $(b)$, Carol $(c)\}$ is the set of players, and $S_{i}=\left\{s_{i}^{1}, s_{i}^{2}\right\}$ the finite set of strategies of each $i \in N$. The payoff functions of Ann and Bob are independent of the opponents' strategy profile, i.e., for each $i \in\{a, b\}$,

$$
U_{i}\left(s_{i}, s_{-i}\right)= \begin{cases}1 & \text { if } s_{i}=s_{i}^{1} \\ 0 & \text { if } s_{i}=s_{i}^{2}\end{cases}
$$

for every $s_{-i} \in S_{-i}$. On the other hand, Carol's payoff function is given by

$$
U_{c}\left(s_{a}, s_{b}, s_{c}\right)= \begin{cases}1 & \text { if }\left(s_{a}, s_{b}, s_{c}\right)=\left(s_{a}^{1}, s_{b}^{1}, s_{c}^{1}\right) \\ 2 & \text { if }\left(s_{a}, s_{b}, s_{c}\right)=\left(s_{a}^{2}, s_{b}^{2}, s_{c}^{2}\right) \\ 0 & \text { otherwise. }\end{cases}
$$

Notice that the only correlated rationalizable strategy profile is $\left(s_{a}^{1}, s_{b}^{1}, s_{c}^{1}\right)$ : Playing $s_{i}^{2}$ is strictly dominated for any Ann and Bob, and therefore they both eliminate it. Then, at the second round of elimination, Carol wipes out $s_{c}^{2}$, as $U_{c}\left(s_{a}^{1}, s_{b}^{1}, s_{c}^{1}\right)>U_{c}\left(s_{a}^{1}, s_{b}^{1}, s_{c}^{2}\right)$.

Now, consider an epistemic model $\mathcal{A}^{\Gamma}$ of $\Gamma$ :

$$
\begin{aligned}
\Omega & =\left\{\omega_{1}, \omega_{2}, \omega_{3}\right\} \text { endowed with a uniform common prior } \pi \\
\mathcal{P}_{a} & =\left\{\left\{\omega_{1}, \omega_{2}\right\}_{s_{a}^{1}} ;\left\{\omega_{3}\right\}_{s_{a}^{2}}\right\}, \\
\mathcal{P}_{b} & =\left\{\left\{\omega_{1}\right\}_{s_{b}^{1}} ;\left\{\omega_{s_{b}^{1}}\right\}_{s_{b}^{1}} ;\left\{\omega_{3}\right\}_{s_{b}^{2}}\right\}, \\
\mathcal{P}_{c} & =\left\{\left\{\omega_{1}\right\}_{s_{c}^{1}} ;\left\{\omega_{2}, \omega_{3}\right\}_{s_{c}^{1}}\right\},
\end{aligned}
$$

with the information sets' indices denoting the respective player's strategy given by the choice function. Let $G=(N, \mathcal{E})$ be a connected graph such that

$$
\begin{aligned}
N & =\{\text { Ann, Bob, Carol }\} \\
\mathcal{E} & =\{(\text { Ann, Bob }),(\text { Bob }, \text { Carol })\} .
\end{aligned}
$$

Firstly, observe that it is $G$-pairwise mutual knowledge at $\omega_{1}$ that players deem possible only strategy profiles belonging to the support of the actual conjectures. More specifically, notice that the states where the players deem possible only strategy profiles that belong to the supports of the conjectures at 
$\omega_{1}$ are

$$
\begin{aligned}
C_{a}\left(\operatorname{Supp}\left(\hat{\mu}_{a}\left(\omega_{1}\right)\right)\right) & =\left\{\omega \in \Omega: \operatorname{Supp}\left(\hat{\mu}_{a}(\omega)\right) \subseteq \operatorname{Supp}\left(\hat{\mu}_{a}\left(\omega_{1}\right)\right)\right\} \\
& \left.=\left\{\omega \in \Omega: \operatorname{Supp}\left(\hat{\mu}_{a}(\omega)\right) \subseteq\left\{\left(s_{b}^{1}, s_{c}^{1}\right)\right\}\right\}\right) \\
& =\left\{\omega_{1}, \omega_{2}\right\}
\end{aligned}
$$

and likewise

$$
\begin{aligned}
& C_{b}\left(\operatorname{Supp}\left(\hat{\mu}_{b}\left(\omega_{1}\right)\right)\right)=\left\{\omega_{1}, \omega_{2}\right\}, \\
& C_{c}\left(\operatorname{Supp}\left(\hat{\mu}_{c}\left(\omega_{1}\right)\right)\right)=\left\{\omega_{1}\right\} .
\end{aligned}
$$

Then, it is straightforward verifying that

$$
\begin{aligned}
\omega_{1} & \in K_{a, b}\left(\left\{\omega_{1}, \omega_{2}\right\}\right) \cap K_{b, c}\left(\left\{\omega_{1}\right\}\right) \\
& =K_{a, b}\left(C_{a}\left(\operatorname{Supp}\left(\hat{\mu}_{a}\left(\omega_{1}\right)\right)\right) \cap C_{b}\left(\operatorname{Supp}\left(\hat{\mu}_{b}\left(\omega_{1}\right)\right)\right)\right) \cap K_{b, c}\left(C_{b}\left(\operatorname{Supp}\left(\hat{\mu}_{b}\left(\omega_{1}\right)\right)\right) \cap C_{c}\left(\operatorname{Supp}\left(\hat{\mu}_{c}\left(\omega_{1}\right)\right)\right)\right) .
\end{aligned}
$$

However, observe that it is not mutually known that $\omega_{1}$ that players deem possible only strategy profiles that receive probability by the conjecture at $\omega_{1}$, as $\omega_{1} \notin K_{a}\left(C_{c}\left(\operatorname{Supp}\left(\hat{\mu}_{c}\left(\omega_{1}\right)\right)\right)\right)$, implying that the second condition of Theorem 1 is violated.

Secondly, note that rationality is $G$-pairwise mutual knowledge at $\omega_{1}$. However, it is not mutually known at $\omega_{1}$ that everyone is rational. Indeed, Ann does not know at $\omega_{1}$ that Carol is rational, as at $\omega_{2}$ Carol's unique best response to her conjecture is to play $s_{c}^{2}$ rather than $s_{c}^{1}$, implying that the first condition of Theorem 1 is not satisfied either.

Finally, observe that

$$
\left(\bigcup_{j \neq a} \operatorname{Proj}_{S_{a}} \operatorname{Supp}\left(\hat{\mu}_{j}\left(\omega_{1}\right)\right)\right) \times\left(\bigcup_{j \neq b} \operatorname{Proj}_{S_{b}} \operatorname{Supp}\left(\hat{\mu}_{j}\left(\omega_{1}\right)\right)\right) \times\left(\bigcup_{j \neq c} \operatorname{Proj}_{S_{c}} \operatorname{Supp}\left(\hat{\mu}_{j}\left(\omega_{1}\right)\right)\right)=\left\{s_{a}^{1}\right\} \times\left\{s_{b}^{1}\right\} \times\left\{s_{c}^{1}\right\}
$$

is the unique rectangle satisfying the best response property, and therefore $\left(\hat{s}_{a}\left(\omega_{1}\right), \hat{s}_{b}\left(\omega_{1}\right), \hat{s}_{c}\left(\omega_{1}\right)\right)=$ $\left(s_{a}^{1}, s_{b}^{1}, s_{c}^{1}\right)$ is a correlated rationalizable strategy profile.

In the preceding example, both the two central elements of Zambrano's sufficient conditions for correlated rationalizability are violated, and yet the conclusion of his theorem does hold. On the basis of this observation, the natural question then arises, whether there exists a general relation between the weaker $G$-pairwise mutual knowledge conditions of Definition 1 on the one hand, and correlated rationalizability on the other hand. This question is answered affirmatively in the next section.

\section{PAIRWISE MUTUAL KNOWLEDGE AND EPISTEMIC CONDITIONS FOR RATIONALIZABILITY}

The following result weakens Zambrano's conditions for correlated rationalizability by means of pairwise mutual knowledge. More specifically, it is shown that $G$-pairwise mutual knowledge of rationality 
and $G$-pairwise mutual knowledge of everybody deeming possible only strategy profiles belonging to the actual support of their conjectures already suffice for correlated rationalizability, if $G$ is connected.

THEOREM 2 Let $\Gamma$ be a normal form game, $\mathcal{A}^{\Gamma}$ an epistemic model of it and $G=(N, \mathcal{E})$ a connected graph. Suppose that there is some state $\omega \in \Omega$ such that $\omega \in K_{i, j}\left(R_{i} \cap R_{j}\right) \cap K_{i, j}\left(C_{i}\left(\operatorname{Supp}\left(\hat{\mu}_{i}(\omega)\right)\right) \cap\right.$ $\left.C_{j}\left(\operatorname{Supp}\left(\hat{\mu}_{j}(\omega)\right)\right)\right)$ for all $(i, j) \in \mathcal{E}$. Then,

$$
\left(\bigcup_{j \neq 1} \operatorname{Proj}_{S_{1}} \operatorname{Supp}\left(\hat{\mu}_{j}(\omega)\right)\right) \times \cdots \times\left(\bigcup_{j \neq n} \operatorname{Proj}_{S_{n}} \operatorname{Supp}\left(\hat{\mu}_{j}(\omega)\right)\right)
$$

satisfies the best response property, and therefore $\left(\hat{s}_{1}(\omega), \ldots, \hat{s}_{n}(\omega)\right)$ is correlated rationalizable.

ProOF: Let $G=(N, \mathcal{E})$ be a connected graph, and suppose for the sake of simplicity and without loss of generality that $(j, j+1) \in \mathcal{E}$ for all $j=1, \ldots, n-1$.

Firstly, we show that $\operatorname{Proj}_{S_{i}} \operatorname{Supp}\left(\hat{\mu}_{j}(\omega)\right)=\operatorname{Proj}_{S_{i}} \operatorname{Supp}\left(\hat{\mu}_{j+1}(\omega)\right)$ for every $i \in N \backslash\{j, j+1\}$. Since $(j, j+1) \in \mathcal{E}$, it follows that $\omega \in K_{j, j+1}\left(C_{j+1}\left(\operatorname{Supp}\left(\hat{\mu}_{j+1}(\omega)\right)\right)\right)$, implying that $\operatorname{Supp}\left(\hat{\mu}_{j+1}\left(\omega^{\prime}\right)\right) \subseteq$ $\operatorname{Supp}\left(\hat{\mu}_{j+1}(\omega)\right)$ for all $\omega^{\prime} \in P_{j}(\omega)$. Therefore, $\operatorname{Proj}_{S_{i}} \operatorname{Supp}\left(\hat{\mu}_{j+1}\left(\omega^{\prime}\right)\right) \subseteq \operatorname{Proj}_{S_{i}} \operatorname{Supp}\left(\hat{\mu}_{j+1}(\omega)\right)$ for all $\omega^{\prime} \in P_{j}(\omega)$. Now, consider some $s_{i} \in \operatorname{Proj}_{S_{i}} \operatorname{Supp}\left(\hat{\mu}_{j}(\omega)\right)$. Then, there is some $\omega^{\prime} \in P_{j}(\omega)$ such that $\hat{s}_{i}\left(\omega^{\prime}\right)=s_{i}$. Hence, $s_{i} \in \operatorname{Proj}_{S_{i}} \operatorname{Supp}\left(\hat{\mu}_{j+1}\left(\omega^{\prime}\right)\right)$, and therefore $s_{i} \in \operatorname{Proj}_{S_{i}} \operatorname{Supp}\left(\hat{\mu}_{j+1}(\omega)\right)$. Thus, we conclude that $\operatorname{Proj}_{S_{i}} \operatorname{Supp}\left(\hat{\mu}_{j}(\omega)\right) \subseteq \operatorname{Proj}_{S_{i}} \operatorname{Supp}\left(\hat{\mu}_{j+1}(\omega)\right)$. Likewise, we show that $\operatorname{Proj}_{S_{i}} \operatorname{Supp}\left(\hat{\mu}_{j+1}(\omega)\right) \subseteq$ $\operatorname{Proj}_{S_{i}} \operatorname{Supp}\left(\hat{\mu}_{j}(\omega)\right)$.

Secondly, we show that for every $s_{i} \in \bigcup_{j \neq i} \operatorname{Proj}_{S_{i}} \operatorname{Supp}\left(\hat{\mu}_{j}(\omega)\right)$ there is some conjecture

$$
\mu_{i} \in \Delta\left(\prod_{k \neq i}\left(\bigcup_{j \neq k} \operatorname{Proj}_{S_{k}} \operatorname{Supp}\left(\hat{\mu}_{j}(\omega)\right)\right)\right)
$$

such that $s_{i} \in B R_{i}\left(\mu_{i}\right)$, which then implies that the rectangle in (1) satisfies the best response property. Since $s_{i} \in \bigcup_{j \neq i} \operatorname{Proj}_{S_{i}} \operatorname{Supp}\left(\hat{\mu}_{j}(\omega)\right)$, there is some $j \neq i$ such that $s_{i} \in \operatorname{Proj}_{S_{i}} \operatorname{Supp}\left(\hat{\mu}_{j}(\omega)\right)$. Without loss of generality assume that $j<i$. Then, it follows from repeatedly applying the previous step that $s_{i} \in$ $\operatorname{Proj}_{S_{i}} \operatorname{Supp}\left(\hat{\mu}_{i-1}(\omega)\right)$. Therefore, there is some $\omega^{\prime \prime} \in P_{i-1}(\omega)$ such that $s_{i}\left(\omega^{\prime \prime}\right)=s_{i}$. Moreover, it follows from $\omega \in K_{i-1, i}\left(R_{i}\right)$ that $s_{i}\left(\omega^{\prime \prime}\right) \in B R_{i}\left(\hat{\mu}_{i}\left(\omega^{\prime \prime}\right)\right)$. Finally, it follows from $\omega \in K_{i-1, i}\left(C_{i}\left(\operatorname{Supp}\left(\hat{\mu}_{i}(\omega)\right)\right)\right)$ that $\operatorname{Supp}\left(\hat{\mu}_{i}\left(\omega^{\prime \prime}\right)\right) \subseteq \operatorname{Supp}\left(\hat{\mu}_{i}(\omega)\right)$. Therefore, since $\operatorname{Supp}\left(\hat{\mu}_{i}(\omega)\right) \subseteq \prod_{k \neq i}\left(\bigcup_{j \neq k} \operatorname{Proj}_{S_{k}} \operatorname{Supp}\left(\hat{\mu}_{j}(\omega)\right)\right)$, it follows that $\hat{\mu}_{i}\left(\omega^{\prime \prime}\right) \in \Delta\left(\prod_{k \neq i}\left(\bigcup_{j \neq k} \operatorname{Proj}_{S_{k}} \operatorname{Supp}\left(\hat{\mu}_{j}(\omega)\right)\right)\right)$, which completes the proof. $\quad$ Q.E.D.

The contribution of the previous result is two fold: Firstly, it significantly weakens the epistemic foundations for correlated rationalizability of Zambrano (2008) by simultaneously relaxing both his conditions. Secondly, this is the first paper in the literature to provide sufficient epistemic conditions for iterated elimination of strictly dominated strategies that do not involve mutual knowledge of rationality. 
Finally, notice that the assumption of the graph being connected is crucial for Theorem 2. In this sense, our epistemic foundation is tight. To see this, recall the game from Example 1, and suppose instead that Bob is connected with Ann, but not with Carol, i.e., $G=(N, \mathcal{E})$ is such that $\mathcal{E}=\{(\mathrm{Ann}, \mathrm{Bob})\}$. Moreover, let Carol play $s_{c}^{2}$ at all states. Then, notice that all conditions of Theorem 2 are satisfied and still the strategy profile played at $\omega_{1}$ is not correlated rationalizable.

\section{Department of Economics, Maastricht University \\ e.tsakas@maastrichtuniversity.nl}

\section{REFERENCES}

Aumann, R.J. (1976). Agreeing to disagree. Annals of Statistics 4, 1236-1239.

Aumann, R.J. \& Brandenburger, A. (1995). Epistemic conditions for Nash equilibrium. Econometrica 63, 1161-1180.

Bach, C.W. \& Tsakas, E. (2012). Pairwise interactive knowledge and Nash equilibrium. Research Memorandum, Maastricht University.

Bernheim, D. (1984). Rationalizable strategic behavior. Econometrica 52, 1007-1028.

Brandenburger, A. \& Dekel, E. (1987). Rationalizability and correlated equilibria. Econometrica 55, 1391-1402.

BöGe, W. \& Eisele, T.H. (1979). On solutions of Bayesian games. International Journal of Game Theory 8, $193-215$.

Pearce, D.G. (1984). Rationalizable strategic behavior and the problem of perfection. Econometrica 52, 1029-1050.

Perea, A. (2012). From classical to epistemic game theory. Research Memorandum, Maastricht University.

Tan, T. \& Werlang, S. (1988). The Bayesian foundations of solution concepts of games. Journal of Economic Theory $45,370-391$.

Zambrano, E. (2008). Epistemic conditions for rationalizability. Games and Economic Behavior 63, 395-405. 